\title{
Invariant Imbedding and Neutron Transport Theory II-Functional Equations
}

\author{
RICHARD BELLMAN, ROBERT KALABA \& \\ G. MILTON WING*
}

\begin{abstract}
SUMMARY
Some one-dimensional fission processes are considered with particular regard to questions of critical length and energy dependence of the products of fission. Various interconnections between the functions used in the invariant imbedding approach and the classical approach are indicated. In particular, it is shown how one may pass from the consideration of certain linear two-point boundary value problems to nonlinear (Riccatian type) initial value problems. The former are useful for theoretical considerations, the latter for numerical computation.
\end{abstract}

\section{INTRODUCTION}

In our two previous papers on the application of the principle of invariant imbedding to neutron transport processes, [3], [4], we considered the problem of determining probability distributions for the reflected and transmitted fluxes associated with one-dimensional rods. Using only expected values, these techniques led to methods for determining critical length.

The customary method of treating these questions, based upon the equation of transfer, leads to linear differential equations for the expected values, with two-point boundary conditions. The critical length is calculated as the solution of an eigenvalue problem.

The method presented in [3] and [4] is of an entirely different nature. The basic equations are now nonlinear differential equations whose solutions are determined solely by initial values. The critical length turns out to be the first value for which the solutions of the equations for expected flux become infinite.

In view of the fact that these two approaches to problems of this type appear to be so disparate, it is of some interest to link them, and to understand the

* Work performed in part under the auspices of the U.S. Atomic Energy Commission. 
relation between the various functions that are introduced in these fashions. In [1], where invariance principles are used to treat the closely related processes of radiative transfer, Ambarzumian states that V. V. Sobolev has obtained various connections between the solutions of the equations derived from invariance principles and the functions arising from the classical equations of mathematical physics. We have been unable to secure these papers, and although we have searched the literature for papers on this topic, we have not been able to find any.

It is this interrelation which we propose to discuss in this paper in some detail. We shall show that the equation of transfer, as well as the functional equations obtained previously, may all be derived from a common point of view. In some cases, this method yields linear equations with two-point boundary conditions; in other cases, it yields nonlinear equations with initial value conditions; in still other cases, we obtain the desired relations connecting the solutions of the two types of equations.

The great analytic utility of linear equations for a rigorous study of the character and nature of diffusion processes must certainly be emphasized, $c f$. Feller, [6], Lehner \& Wing, [9]. Nonetheless, in connection with the computational determination of the solution, two-point boundary conditions cause serious difficulties, $c f$., for example, Davison, [5], for a discussion of a number of methods utilized to treat these problems. The nonlinear equations, although certainly not as handy for analytic purposes, possess the merit of requiring only initial conditions for their numerical solution. They seem well suited, in consequence, for modern digital computers. For references to related work of REDHEFFER and LUNEBERG, see [2]. In connection with the transmission line equations, the paper of PiERce, [10], is relevant.

Any rigorous discussion of the stochastic processes we consider here would take us too far afield. We refer the reader interested in these matters to the forthcoming monograph by T. E. HARRIs, [8], and to his expository paper, [7]. Similarly, to avoid digressions which would divert us from our principal object, that of illustrating the applicability of a variety of functional equation techniques, we shall, in the main, consider only expected fluxes. To determine full probability distributions would require for the general case a study of characteristic functionals, a topic of some complexity. Only in the time-andenergy-independent case, do we discuss the complete distribution.

We shall consider only one-dimensional processes, treating in turn the timeindependent, energy-independent case; the time-dependent, energy-independent case; and the time-independent, energy-dependent case. In the study of the time-dependent case, we are grateful to T. E. HARRIs for the suggestion to introduce moment density functions and for much other helpful criticism.*

*See M. BartLett, An Introduction to Stochastic Processes, pp. 78-83, where many references to the work of BHABHA and Ramakrishnan may be found.

A list of definitions of variables is given at the end of the paper. 
1. A Mathematical Model of One-Dimensional Neutron Transport. Let us now define what we shall mean by a one-dimensional neutron transport process. A neutron is considered to be a point moving on a line interval until an event occurs which causes it to transform into two neutrons of similar nature, one moving to the left and one to the right. This event will be called fission and the process called a fission process.

The probability that a neutron will undergo fission in an infinitestimal interval of length $y$ is taken to be $\lambda^{-1} y+o(y)$, and the probability that it can traverse an interval $y$ without this occurring is $1-\lambda^{-1} y+o(y)$.

Here $\lambda$ represents a "mean free path". We have adjusted our notation to conform to that current in physical circles.

The process can be complicated by the assumption of inhomogeneity, i.e., $\lambda^{-1} y$ replaced by the function $1 / \lambda(y)$; by the introduction of a distribution function for the number and direction of the particles produced by fission; and by taking account of absorption and collisions which merely reverse direction without inducing fission. Since these more realistic features merely add arithmetic complexity without analytic insight, we have ignored their existence in this preliminary treatment.

We shall, however, discuss the case in which the neutron and its progeny posess energies which affect, and are affected by, fission.

2. Invariance Principles. As we have explained in earlier papers, cf. [3], [4], our aim is to introduce certain parameters with the property that the neutron transport process described above can be considered as a sequence of stochastic processes which induce transformations of these parameters. Furthermore, it is desirable to have these parameters represent physically meaningful quantities such as time, energy, length, and so on, essentially quantities which correspond to measurables and observables. This is merely desirable, but should not be insisted upon to the detriment of the analysis. That there are no indispensable variables is quite evident by now.

In applying invariance principles, we can borrow some terminology from communication theory and consider each physical process to consist of three principal elements, a source, an observer, and a transmitting medium or communication channel. As we shall see below, the classical equations of mathematical physics, of which the equation of transfer is typical, arise when functional equation techniques are applied to processes defined in terms of variables describing the observer, or the source. On the other hand, the new equations of invariant imbedding arise when the process is described partly in terms of the medium and partly in terms of the observer or source.

By describing a single process partly in one set of terms and partly in other terminology, we shall obtain the desired equations linking the various formulations. There is much to be done in this direction, with this paper merely a single step in the direction of a more comprehensive theory. We shall restrict ourselves here to one-dimensional processes. 


\section{Time-Independent, Energy-Independent Case}

3. First Formulation. We first recall some results derived in [3].

Consider a finite, homogeneous rod, the interval from $x$ to 0 as indicated below.

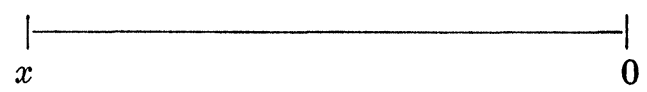

Figure 1

Let us start with the case where a "trigger" neutron enters the rod from the left, at $x$, at time zero. We shall assume that energy is neglected, and rid ourselves of time considerations by asking for the respective probabilities that precisely $n$ neutrons are reflected from the rod (which is to say, emerge from $x$ to the left), over all time, for $n=0,1,2, \cdots$.

This probability will be denoted by $p(n, x)$. In introducing this notation, in place of what customarily might be called $p(n)$, we are deliberately calling attention to the fact that the length of the rod is to be treated in this section as an essential parameter of the process.

We are thus imbedding this particular process in the family of processes of similar nature in which the rod can assume any positive length.

Using invariance principles, as described in [4] in detail, we obtain the following system of nonlinear differential equations:

$$
\begin{aligned}
& p^{\prime}(0, x)=-\lambda^{-1} p(0, x) \\
& \begin{aligned}
p^{\prime}(n, x)=-(n+1) \lambda^{-1} p(n, x)+\lambda^{-1} p(n & -1, x) \\
& +\lambda^{-1} \sum_{k=1}^{n} k p(k, x) p(n-k, x),
\end{aligned}
\end{aligned}
$$

valid for $x \geqq 0$, along with the initial condition $p(m, 0)=\delta_{0 m}$.

The study of these equations is, as usual, greatly facilitated by the introduction of the generating function

$$
h(x, r)=\sum_{n=0}^{\infty} p(n, x) r^{n}
$$

Using the equations in (3.1), we readily see that $h(x, r)$ satisfies the quasilinear partial differential equations

$$
h_{x}=\lambda^{-1}(r-1) h+\lambda^{-1} r(h-1) h_{r} .
$$

Either from this equation, or directly from (3.1) (also see Section 7), we derive the Riccati equation

$$
U^{\prime}=\lambda^{-1}\left(1+U^{2}\right)
$$

for the expected number $U(x)$ of reflected neutrons. This equation is valid for $0 \leqq x \leqq x_{c}$, where $x_{c}$ is the critical length which turns out to be $\frac{1}{2} \pi \lambda$. 
As we shall see below, this equation can be derived from first principles, without the introduction of higher moments, or use of the generating function. On the other hand, it is also possible to obtain the partial differential equation for the generating function by these direct techniques.

4. Second Formulation. In this formulation of a fission process, we begin with a particle situated at a point $y$, interior to the rod, at time zero and starting off either to the left or to the right.

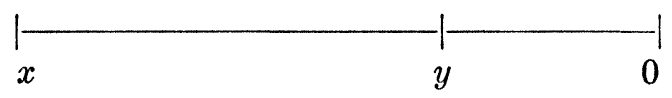

FIGURe 2

Let us define the probabilities

$$
\begin{aligned}
q_{L}(n, y)= & \text { the probability that a particle starting to the left at } y \text { at } \\
& \text { time zero will result in } n \text { particles emerging from } x \text { over } \\
& \text { all time, } \\
q_{R}(n, y)= & \text { the same probability assuming that the particle starts to } \\
& \text { the right at the initial instant. }
\end{aligned}
$$

Observe that we have now suppressed the $x$-dependence, indicating that we wish to keep $x$ fixed throughout the ensuing analysis, and have focused our attention upon $y$. We wish to imbed this process within the family of processes of this type in which $y$ takes on any value between 0 and $x$.

To obtain equations characterizing the function we have just introduced, we follow the path of the particle from $y$ to $y \pm \Delta$, dependent upon the initial direction. If, for example, the particle suffers a collision in $[y, y-\Delta]$, we obtain a new process in which one particle starts from the right at $y-\Delta$ and the other starts from the left at $y-\Delta$; if there is no collision, we are left with a process of the same type for an initial particle at $y-\Delta$ starting to the right.

Taking account of these possibilities, it is easy to see that we obtain the relations

$$
\begin{aligned}
& q_{L}(0, y)=0, \\
& \begin{aligned}
& q_{L}(n, y)=\lambda^{-1} \Delta\left[\sum_{k=1}^{n} q_{L}(k, y) q_{R}(n-k, y)\right] \\
&+\left(1-\lambda^{-1} \Delta\right) q_{L}(n, y+\Delta)+o(\Delta),
\end{aligned}
\end{aligned}
$$

for $0<y \leqq x$, and $n=1,2, \cdots$, with the boundary conditions

$$
q_{L}(n, x)=\delta_{1 \mathrm{n}} .
$$

We obtain a similar set of recursion relations for the sequence $\left\{q_{R}(n, y)\right\}$ with the boundary conditions

$$
q_{R}(n, 0)=\delta_{0 n} .
$$


From these approximate relations, we derive the following nonlinear differential equations:

$$
\begin{aligned}
& q_{L}^{\prime}(n, y)=\lambda^{-1} q_{L}(n, y)-\lambda^{-1} \sum_{l=1}^{n} q_{L}(l, y) q_{R}(n-l, y), \\
& q_{R}^{\prime}(n, y)=-\lambda^{-1} q_{R}(n, y)+\lambda^{-1} \sum_{l=0}^{n} q_{R}(l, y) q_{L}(n-l, y),
\end{aligned}
$$

for $0<y<x$.

The generating functions, defined by the series

$$
f(r, y)=\sum_{n=0}^{\infty} q_{L}(n, y) r^{n}, \quad g(r, y)=\sum_{n=0}^{\infty} q_{R}(n, y) r^{n},
$$

satisfy the nonlinear partial differential equations and boundary conditions

$$
\begin{gathered}
f_{\nu}=-\lambda^{-1} f(r, y) g(r, y)+\lambda^{-1} f(r, y), \\
g_{\nu}=\lambda^{-1} f(r, y) g(r, y)-\lambda^{-1} g(r, y), \\
f(r, x)=r, \quad g(r, 0)=1 .
\end{gathered}
$$

What is amazing, and a great advantage of this formulation, is that the moments $\sum_{n=1}^{\infty} n^{k} q_{L}(n, y)$ and $\sum_{n=1}^{\infty} n^{k} q_{R}(n, y), k=1,2, \cdots$, satisfy linear differential equations. Introducing the expected values

$$
u_{R}(y)=\sum_{n=1}^{\infty} n q_{R}(n, y), \quad u_{L}(y)=\sum_{n=1}^{\infty} n q_{L}(n, y),
$$

we obtain, through differentiation of equation (4.7), the linear system of differential equations

$$
\begin{aligned}
& u_{R}^{\prime}(y)=\lambda^{-1} u_{L}(y), \\
& u_{L}^{\prime}(y)=-\lambda^{-1} u_{R}(y), \quad 0<y<x .
\end{aligned}
$$

The boundary conditions are now two-point:

$$
u_{R}(0)=0, \quad u_{L}(x)=1 .
$$

5. Critical Mass. One of the principal aims of a theory of neutron transport is the determination of critical mass. We equate the physical phenomenon of criticality with the mathematical concept of singularity. In both analytic approaches, we seek a length of rod which results in an infinite expected value, cf. [3] and [4].

The details of the analysis, however, are quite different in the two formulations.

In the first approach, we have an ordinary differential equation of Riccati type, with a given initial value, which we integrate numerically point-by-point until the solution blows up. The first critical point encountered is the required critical length. 
In the second approach, we solve a system of linear differential equations with a two-point boundary condition. This method is closely allied to classical eigenvalue techniques, with the length $x$ playing the role of an eigenvalue.

In the simple time-independent, energy-independent case treated above, both methods are readily pursued, both yielding explicit analytic expressions. It is only when we turn to the discussion of more realistic processes that a fork in the road develops. A formulation which yields linear equations appears very much more desirable if a theoretical treatment is contemplated, while an approach based upon nonlinear equations determined by initial conditions appears preferable if a numerical treatment is desired.

In future work, we shall discuss these points in greater detail.

6. Interlinking of the Two Techniques. Having seen that two quite different analytic approaches to the study of neutron transport are possible, we wish to determine the relations, if any, that exist among the various functions we have defined. This linking of techniques can be useful in two ways. On the one hand, it shows us how to linearize certain types of Riccatian functional equations, and, on the other hand, it shows how to replace two-point boundary value problems involving linear equations by initial value problems for nonlinear equations.

In order to obtain the desired relations, we combine the two formulations in the following manner. In calculating $q_{R}(n, y)$, we first observe that as a result of one neutron entering $[y, 0]$ from the left, which is to say leaving $[x, y]$ from the right, we have a probability $p(k, y)$, in the notation of Section 3 , of having $k$ neutrons reflected. Here we are speaking only of those neutrons that emerge from $[y, 0]$, moving to the left, for the first time. Each of these $k$ neutrons can now be considered as the initiator of a new process starting from $y$ in which a neutron enters $[x, y]$ from the right, the particle moving to the left.

In these terms, let us see how we can obtain exactly $n$ neutrons emerging from $x$ over all time. Suppose that the first of the neutrons described above produces $n_{1}$ neutrons, the second $n_{2}$, and the $k^{\text {th }}$ a quantity $n_{k}$, where

$$
n_{1}+n_{2}+\cdots n_{k}=n \text {. }
$$

Since the probability of this event is

$$
q_{L}\left(n_{1}, y\right) q_{L}\left(n_{2}, y\right) \cdots q_{L}\left(n_{k}, y\right),
$$

we derive the relation

$$
q_{R}(n, y)=\sum_{k=1}^{\infty} p(k, y)\left[\sum q_{L}\left(n_{1}, y\right) q_{L}\left(n_{2}, y\right) \cdots q_{L}\left(n_{k}, y\right)\right],
$$

where the inner summation is taken over the lattice-points satisfying equation (6.1).

Recalling the definitions 


$$
\begin{aligned}
& \sum_{n=0} q_{R}(n, y) r^{n}=g(r, y), \\
& \sum_{n=0} q_{L}(n, y) r^{n}=f(r, y), \\
& \sum_{n=0} p(n, x) r^{n}=h(x, r),
\end{aligned}
$$

we obtain from equation (6.3) a relation connecting the generating functions

$$
g(r, y)=h(x, f(r, y))
$$

An analogous relation can be derived if we consider $q_{L}(y, r)$ initially.

From these relations we can derive equations connecting the expected values. However, as we shall see below, these equations can readily be obtained directly.

The remainder of the paper will be devoted solely to a discussion of expected values, since the analogues of the results above for generating functions are now relations connecting characteristic functionals. Since consideration of these entities would both complicate the analysis and elevate the conceptual level, we have kept them offstage until another date.

7. Expected Values. Let us now indicate how to obtain the equations for expected values without passing through the intermediate step of probability distributions. This discussion is preparatory to the course we shall pursue in the treatment of the more complicated time-dependent and energy-dependent cases. We may think of the processes as being deterministic, as is common in radiative transfer theory.

Let $U(x)$ denote the expected number of neutrons reflected from $[x, 0]$ over all time as the result of an incident trigger neutron at time zero. In passing through the segment $[x, x-\Delta]$ the trigger neutron may undergo fission, in which case one neutron is reflected and one is incident on $[x-\Delta, 0]$, the expected number of reflected neutrons from this segment being $U(x-\Delta)$. If the trigger neutron does not undergo fission, then the expected number of neutrons reflected directly is $U(x-\Delta)$. In addition, one of the neutrons reflected from the segment $[x-\Delta, 0]$ may undergo fission giving rise to another trigger neutron incident on the segment $[x-\Delta, 0]$, which in turn gives rise to the expected number of reflected neutrons $U(x-\Delta)$. All other processes lead to terms of at least second order in $\Delta$. Taking account of these possibilities, we obtain the equation

$$
\begin{aligned}
U(x)=\lambda^{-1} \Delta(1+U(x & -\Delta))+\left(1-\lambda^{-1} \Delta\right) U(x-\Delta) \\
& +\left(1-\lambda^{-1} \Delta\right) U(x-\Delta) \lambda^{-1} \Delta U(x-\Delta)+o(\Delta) .
\end{aligned}
$$

Letting $\Delta \rightarrow 0$, we obtain the differential equation

$$
U^{\prime}(x)=\lambda^{-1}\left[1+U^{2}(x)\right], \quad U(0)=0 .
$$

In addition, if we let 
$V(x)=$ the expected number of neutrons emergent from one end of a rod of length $x$ as a result of a trigger neutron incident at the other end, we find that $V(x)$ satisfies the equation

$$
V^{\prime}(x)=\lambda^{-1} U(x) V(x), \quad V(0)=1 .
$$

Similarly, we see that $u_{R}(y)$ and $u_{L}(y)$ satisfy the linear equations

$$
\begin{array}{ll}
u_{R}^{\prime}(y)=\lambda^{-1} u_{L}(y), & u_{R}(0)=0, \\
u_{L}^{\prime}(y)=-\lambda^{-1} u_{R}(y), & u_{L}(x)=1 .
\end{array}
$$

To derive equations (7.4) we trace the trigger neutron through either the segment $[y+\Delta, y]$ or $[y, y-\Delta]$, as the case may be. Considering the case in which it moves to the right, we find that if it does not undergo fission on the segment $[y+\Delta, y]$, we have, in effect, a process in which there is a trigger neutron moving to the right at $y$. If it does undergo fission then we have a process in which there are trigger neutrons moving toward the right and toward the left at $y$. Taking into account the probabilities of these events we have

$$
u_{R}(y+\Delta)=\left(1-\lambda^{-1} \Delta\right) u_{R}(y)+\lambda^{-1} \Delta\left\{u_{R}(y)+u_{L}(y)\right\}+o(\Delta) .
$$

Similarly, for $u_{L}(y)$ we find

$$
u_{L}(y)=\left(1-\lambda^{-1} \Delta\right) u_{L}(y+\Delta)+\lambda^{-1} \Delta\left\{u_{R}(y+\Delta)+u_{L}(y+\Delta)\right\}+o(\Delta) .
$$

Equations (7.4) follow directly from these.

Following the argument given in the previous section, it is easy to see that we obtain the following two equations:

$$
\begin{aligned}
& u_{R}(y)=U(y) u_{L}(y), \\
& u_{L}(y)=V(x-y)+U(x-y) u_{R}(y), \quad 0 \leqq y \leqq x<x_{c},
\end{aligned}
$$

which connect the solutions to the linear and nonlinear differential equations given above.

Consequently the transformation formulas are

$$
\begin{aligned}
& u_{R}(y)=\frac{U(y) V(x-y)}{1-U(y) U(x-y)}, \\
& u_{L}(y)=\frac{V(x-y)}{1-U(y) U(x-y)},
\end{aligned}
$$

and

$$
\begin{aligned}
U(y) & =\frac{u_{R}(y)}{u_{L}(y)}, \\
V(x-y) & =u_{L}(y)-u_{R}(y) \frac{u_{R}(x-y)}{u_{L}(x-y)} .
\end{aligned}
$$


By means of these relations, we can pass from the solution of one type of equation to the other. We have thus obtained a set of relations linking the two types of functions.*

\section{Time-and-Energy-Independent, Variable Observer. Let}

$$
\begin{aligned}
w_{R}(z ; x)= & \text { the expected number of neutrons passing } z \text { toward the } \\
& \text { right over all time as a result of one trigger neutron entering } \\
& \text { the bar }[x, 0] \text { at the left, at } x, \\
w_{L}(z ; x)= & \text { the corresponding number passing to the left. }
\end{aligned}
$$

Then we easily derive the equations

$$
\begin{aligned}
& w_{R}(z+\Delta ; x)=w_{R}(z ; x)-w_{L}(z ; x) \lambda^{-1} \Delta+o(\Delta), \\
& w_{L}(z+\Delta ; x)=w_{L}(z ; x)+w_{R}(z ; x) \lambda^{-1} \Delta+o(\Delta),
\end{aligned}
$$

which lead to the linear system

$$
\begin{aligned}
& w_{R}^{\prime}(z ; x)=-\lambda^{-1} w_{L}(z ; x), \\
& w_{L}^{\prime}(z ; x)=\lambda^{-1} w_{R}(z ; x), \quad 0<z \leqq x,
\end{aligned}
$$

where the primes denote differentiation with respect to $z$. The two-point boundary conditions are

$$
w_{R}(x ; x)=1, \quad w_{L}(0 ; x)=0 .
$$

Comparing these equations and boundary conditions with those obtained for $u_{R}$ and $u_{L}$ in the previous section, we find that

$$
u_{L}(z)=w_{R}(z ; x), \quad u_{R}(z)=w_{L}(z ; x)
$$

This system constitutes a reciprocity law which states, in part, that the expected number of neutrons emerging from $x$, over all time, as the result of a trigger neutron moving to the left at $z$ is equal to the expected number of neutrons, over all time, moving to the right at $z$ as a result of a trigger neutron introduced at $x$. The reciprocity obviously holds under much more general circumstances.

9. The Internal Flux. Let us now consider the expected number of neutrons moving to the right which pass a fixed point $z$ as a result of a source neutron moving to the right at a fixed point $y, y>z$ (see Fig. 3). We consider this as a function of the length $x$ of the bar, say $f(x ; y, z), y$ and $z$ being held fixed. This will illustrate

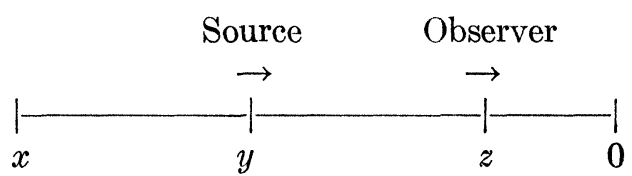

Frgure 3

*When the differential equations are solved explicitly, these relations reduce to trigonometrical identities. 
how internal flows with arbitrarily placed sources may be determined. If $x=y$, then

$$
f(x ; y, z)=f(y ; y, t)=w_{R}(z ; y)
$$

in the notation of the previous section. If $x>y$, then

$$
f(x ; y, z)=w_{R}(z ; y)+U(y) U(x-y) w_{R}(z ; y) .
$$

The first term in the right hand side of the above equation is the direct flux and the second is the secondary flux due to reflections from the segments $[y, 0]$ and $[x, y]$.

\section{Time-Dependent, Energy-Independent Case}

10. Expected Value. Let us now consider a time-dependent process. It was pointed out to the authors by T. E. HARRIs that the techniques used in the preceding sections can be equally well employed in the discussion of timedependence, provided that we introduce the important moment density function.

We define the following two functions:

$$
\begin{aligned}
U(x, t)= & \text { the expected number of particles emerging from } x \\
& \text { between time zero and time } t \text { from a rod of length } x \text { due } \\
& \text { to a trigger neutron entering at } x \text { at time zero. } \\
= & \text { the expected number between } t \text { and } t+d t \text { arising in } \\
& \text { the same manner. }
\end{aligned}
$$

Note that $U_{t}=u$.

To obtain an equation for $U(x, t)$, we begin with the fact that a neutron traversing $[x+\Delta, x]$ splits with probability $\lambda^{-1} \Delta$ and continues unaltered with probability $\left(1-\lambda^{-1} \Delta\right)$. The neutron which has not suffered a collision produces a certain flux of neutrons from $[x, 0]$ in the time interval $[s, s+d s]$, namely $u(x, s) d s$. The velocity of a neutron is denoted by $c$.

Of this flux, an expected amount $\left(1-\lambda^{-1} \Delta\right) u(x, s) d s$ continues through $[x+\Delta, x]$ without collision, while $\lambda^{-1} \Delta u(x, s) d s$ produces neutrons going in both directions. The quantity $\lambda^{-1} \Delta u(x, s) d s$ going to the right produces an expected reflection of $U(x, t-s) \cdot \lambda^{-1} \Delta u(x, s) d s$, during the remaining time.

Adding these effects together, we see that

$$
\begin{aligned}
U(x & \left.+\Delta, t+\frac{2 \Delta}{c}\right)=\lambda^{-1} \Delta[U(x, t)+1] \\
& +\left(1-\lambda^{-1} \Delta\right)\left\{U(x, t)+\int_{0}^{t} u(x, s) \lambda^{-1} \Delta U(x, t-s) d s\right\}+o(\Delta) .
\end{aligned}
$$

Passing to the limit, we obtain the integro-differential equation

$$
U_{x}+\frac{2}{c} U_{t}=\lambda^{-1} \int_{0}^{t} u(x, s) U(x, t-s) d s+\lambda^{-1} .
$$


The initial conditions in space and time are

$$
U(x, 0)=0, \quad U(0, t)=0 .
$$

Using Laplace transform techniques, we can solve for $U$ since the equation in (10.3) is of convolution type. A number of interesting questions arise in this analysis which we shall discuss at a later date.

Let

$$
\begin{aligned}
V(x, t)= & \text { the expected number of neutrons transmitted through a } \\
& \text { bar of length } x \text { in time } t .
\end{aligned}
$$

Then we obtain the equation

$$
\begin{aligned}
& V\left(x+\Delta, t+\frac{\Delta}{c}\right) \\
& \quad=V(x, t)+\left(1-\lambda^{-1} \Delta\right) \int_{0}^{t} u(x, s) \lambda^{-1} \Delta V(x, t-s) d s+o(\Delta)
\end{aligned}
$$

which leads to the integro-differential equation

$$
V_{x}+\frac{1}{c} V_{t}=\lambda^{-1} \int_{0}^{t} u(x, s) V(x, t-s) d s .
$$

In addition, $V$ satisfies the conditions

$$
V(0, t)=1, \quad V(x, 0)=0 .
$$

11. Moving Source. Let us now describe the process in terms of a variable source. Let

$$
\begin{aligned}
u_{L}(y, t)= & \text { the expected number of neutrons emerging from } x \text { over } \\
& \text { the time interval }[0, t] \text { due to an initial neutron moving } \\
& \text { to the left at } y \text { at time zero, } \\
u_{R}(y, t)= & \text { the corresponding quantity due to a neutron moving to } \\
& \text { the right. }
\end{aligned}
$$

Then we readily obtain the following equations:

$$
\begin{gathered}
u_{L}(y, t)=\lambda^{-1} \Delta\left\{u_{L}\left(y+\Delta, t-\frac{\Delta}{c}\right)+u_{R}\left(y+\Delta, t-\frac{\Delta}{c}\right)\right\} \\
+\left(1-\lambda^{-1} \Delta\right) u_{L}\left(y+\Delta, t-\frac{\Delta}{c}\right)+o(\Delta), \\
u_{R}(y, t)=\lambda^{-1} \Delta\left\{u_{R}\left(y-\Delta, t-\frac{\Delta}{c}\right)+u_{L}\left(y-\Delta, t-\frac{\Delta}{c}\right)\right\} \\
+\left(1-\lambda^{-1} \Delta\right) u_{R}\left(y-\Delta, t-\frac{\Delta}{c}\right)+o(\Delta),
\end{gathered}
$$


from whence we obtain the system of differential equations

$$
\begin{aligned}
& \frac{1}{c} \frac{\partial u_{L}}{\partial t}-\frac{\partial u_{L}}{\partial y}=\lambda^{-1} u_{R}, \\
& \frac{1}{c} \frac{\partial u_{R}}{\partial t}+\frac{\partial u_{R}}{\partial y}=\lambda^{-1} u_{L},
\end{aligned}
$$

for $0<y<x, 0<t$, with the conditions

$$
\begin{array}{ll}
u_{L}(y, 0)=0, & u_{L}(x, t)=1, \\
u_{R}(y, 0)=0, & u_{R}(0, t)=0 .
\end{array}
$$

Proceeding as in the time-independent case we readily obtain the following relations, showing the interconnections:

$$
\begin{gathered}
u_{R}(y, t)=\int_{0}^{t} u(y, s) u_{L}(y, t-s) d s \\
u_{L}(y, t)=V(x-y, t)+\int_{0}^{t} u(x-y, s) u_{R}(y, t-s) d s . \\
\text { IV. Time-Independent, Energy-Dependent Case }
\end{gathered}
$$

12. Expected Values. Let us now consider the case where we take into account the fact that the velocity, or energy, of the particle determines the probability of fission. Furthermore, fission will produce two particles with energies different, in general, from that of the parent. For convenience, 0 and $x$ in Fig. 1 are interchanged:

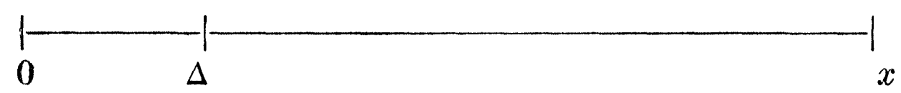

FIGURE 4

We define the functions

$$
\begin{aligned}
u(x, w ; \mathrm{r}) d r= & \text { the expected number of neutrons reflected from } 0 \\
& \text { with energy between } r \text { and } r+d r \text {, over all time, due } \\
& \text { to an initial neutron of energy } w \text { entering the rod at } \\
& 0 \text { at time zero, }
\end{aligned}
$$

(12.1) $v(x, w ; r) d r=$ the expected number transmitted,

$q(s, r) d r \quad=$ the probability of energy change from level $s$ to a level between $r$ and $r+d r$ due to a fissioning,

$\begin{aligned} \frac{\Delta}{\lambda(w)}+o(\Delta)= & \text { the probability of a fissioning in a segment of length } \\ & \Delta \text { of a neutron at energy level } w .\end{aligned}$ 
We then obtain

$$
\begin{aligned}
u(x, w ; r) & =\left(1-\frac{\Delta}{\lambda(w)}\right) u(x-\Delta, w ; r)\left(1-\frac{\Delta}{\lambda(r)}\right) \\
+ & \left(1-\frac{\Delta}{\lambda(w)}\right) \Delta \int_{0}^{\infty} u(x-\Delta, w ; s) q(s, r) \frac{d s}{\lambda(s)} \\
+ & \left(1-\frac{\Delta}{\lambda(w)}\right) \Delta \int_{0}^{\infty} \int_{0}^{\infty} q(s, t) u(x-\Delta, w ; s) u(x-\Delta, t ; r) \frac{d s}{\lambda(s)} d t \\
+ & \frac{\Delta}{\lambda(w)}\left[q(w, r)+\int_{0}^{\infty} u(x, z ; r) q(w, z) d z\right] .
\end{aligned}
$$

Passing to the limit, we obtain the integro-differential equation

$$
\begin{aligned}
\frac{\partial u}{\partial x}=-\left(\frac{1}{\lambda(w)}+\frac{1}{\lambda(r)}\right) u+ & \int_{0}^{\infty} u(x, w ; s) q(s, r) \frac{d s}{\lambda(s)} \\
& +\int_{0}^{\infty} \int_{0}^{\infty} q(s, t) u(x, w ; s) u(x, t ; r) \frac{d s d t}{\lambda(s)} \\
& +\frac{q(w, r)}{\lambda(w)}+\frac{1}{\lambda(w)} \int_{0}^{\infty} u(x, z ; r) q(w, z) d z
\end{aligned}
$$

There is a similar equation for $v(x, w ; r)$, the transmitted flux.

Let us now obtain the functions which will permit us to linearize these equations. Introduce the functions

$$
\begin{aligned}
u_{R}(y, w ; r) d r= & \text { the expected number of neutrons emerging from } 0 \\
& \text { with energy between } r \text { and } r+d r \text { over all time due } \\
& \text { to an initial neutron of energy } w \text { starting to the right } \\
& \text { at } y, 0 \leqq y \leqq x, \text { at time zero, } \\
u_{L}(y, w ; r) d r= & \text { the corresponding quantity when the neutron starts } \\
& \text { to the left. }
\end{aligned}
$$

Then, as before,

$$
\begin{aligned}
& u_{L}(y, w ; r)=\left(1-\frac{\Delta}{\lambda(w)}\right) u_{L}(y-\Delta, w ; r) \\
& +\frac{\Delta}{\lambda(w)}\left[\int_{0}^{\infty} u_{R}(y, s ; r) q(w, s) d s+\int_{0}^{\infty} u_{L}(y, s ; r) q(w, s) d s\right]+o(\Delta),
\end{aligned}
$$

with a corresponding equation for $u_{R}(y, w ; r)$.

To obtain equations connecting these functions, we again proceed as in the previous sections. The result is

$$
\begin{aligned}
& u_{R}(y, w ; r)=\int_{0}^{\infty} u(x-y, w ; s) \cdot u_{L}(y, s ; r) d s \\
& u_{L}(y, w ; r)=v(y, w ; r)+\int_{0}^{\infty} u(y, w ; s) u_{R}(y, s ; r) d s .
\end{aligned}
$$




\section{Definitions of Variables}

$$
\begin{aligned}
& p(n, x)=\text { the probability that } n \text { neutrons are reflected over all time by a } \\
& \text { rod of length } x \text { as a result of an incident neutron. } \\
& h(x, r)=\sum_{n=0}^{\infty} p(n, x) r^{n} . \\
& U(x)=\sum_{n=0}^{\infty} n p(n, x) . \\
& q_{L}(n, y)=\text { the probability that a particle starting to the left at } y \text { at time } \\
& q_{R}(n, y)=\text { the same probability assuming that the particle starts to the } \\
& f(r, y)=\sum_{n=0}^{\infty} q_{L}(n, y) r^{n} . \\
& g(r, y)=\sum_{n=0}^{\infty} q_{R}(n, y) r^{n} . \\
& u_{R}(y)=\sum_{n=1}^{\infty} n q_{R}(n, y) . \\
& u_{L}(y)=\sum_{n=1}^{\infty} n q_{L}(n, y) . \\
& V(x)=\text { the expected number of neutrons emergent from one end of a }
\end{aligned}
$$


$u_{R}(y, t)=$ the corresponding number due to a neutron moving to the right.

$x_{c}=$ the critical length of the bar.

$u(x, w ; r) d r=$ the expected number of neutrons reflected from 0 with energy between $r$ and $r+d r$, over all time, due to an initial neutron of energy $w$ entering the rod at 0 at time zero.

$v(x, w ; r) d r=$ the expected number transmitted.

$q(s, r) d r=$ the probability of energy change from level $s$ to a level between $r$ and $r+d r$ due to a fissioning.

$u_{R}(y, w ; r) d r=$ the expected number of neutrons emerging from 0 with energy between $r$ and $r+d r$, over all time, due to an initial neutron of energy $w$ starting to the right at $y$ at time zero.

$u_{L}(y, w ; r) d r=$ the corresponding quantity when the neutron starts to the left.

\section{REFERENCES}

[1] V. Ambarzumian, et al., Teoreticheskaia Astrofizika, Moscow, 1952 (especially Section 33).

[2] R. Bellman \& R. Kalaba, On the Principle of Invariant Imbedding and Prepagation through Inhomogeneous Media, Proc. Nat. Acad. Sci. U.S.A., 42 (1956), pp. 629-632.

[3] R. Bellman, R. Kalaba \& G. M. Wing, On the Principle of Invariant Imbedding and One-dimensional Neutron Multiplication, Proc. Nat. Acad. Sci. U.S.A., 43 (1957), pp. $517-520$.

[4] R. Bellman, R. Kalaba \& G. M. Wing, On the Principle of Invariant Imbedding and Neutron Transport Theory-I-One-dimensional Case, J. of Math. and Mech., 7 (1958), pp. $149-162$.

[5] B. Davison, Neutron Transport Theory, Clarendon Press, Oxford, 1957.

[6] W. Felder, The General Diffusion Operator and Positivity Preserving Semi-Groups in One Dimension, Ann. of Math., 60 (1954), pp. 417-436.

[7] T. E. HArris, Some Mathematical Models for Branching Processes, Proceedings of the Second Berkeley Symposium on Mathematical Statistics and Probability, Univ. of Cal. Press, 1951.

[8] T. E. Harris, The Theory of Multiplicative Processes, to appear.

[9] J. Lehner \& G. M. Wing, Solution of the Linearized Boltzmann Transport Equation for the Slab Geometry, Duke Math. Jour., 23 (1956), pp. 125-142.

[10] J. R. Pierce, A Note on the Transmission Line Equation in Terms of Impedance, The Bell System Tech. J., 22 (1943), pp. 263-265.

The RAND Corporation

Santa Monica, California and

Los Alamos Scientific Laboratory

Los Alamos, New Mexico 